\title{
Biomarcadores de exposição a chumbo
}

\section{Biomarkers of exposure to lead}

\author{
Raquel Fernanda Gerlach ${ }^{1}$, Soraya Cheier Dibb Gonçalves², Carolina de Souza Guerra ${ }^{3}$
}

\begin{abstract}
RESUMO
O objetivo deste artigo é descrever alguns dos principais biomarcadores de exposição a chumbo, mostrando suas vantagens e limitações, assim como sua relação com exposição presente, passada ou cumulativa. Essas noções básicas são fundamentais para a compreensão dos efeitos do chumbo, da dificuldade do diagnóstico e do problema da exposição a baixas doses. Os biomarcadores de exposição a chumbo mais discutidos neste artigo são sangue, osso e dentes.
\end{abstract}

Palavras-chave: Chumbo. Biomarcadores Farmacológicos. Sangue Total. Osso. Dente.

$\mathrm{O}$ biomarcadores de exposição a chumbo descritos na literatura são sangue, plasma, urina, fezes, saliva, osso, dentes, unhas e cabelo. ${ }^{1,2}$

Há poucos trabalhos sobre medidas de chumbo em cabelos e unhas, e uma quantidade ainda pequena de trabalhos sobre chumbo no plasma e na saliva, embora estes últimos sejam biomarcadores muito promissores. Medidas de chumbo nas fezes não são largamente utilizadas, mas tem papel importante para determinar o grau de exposição pela via gastrointestinal. As medidas de chumbo na urina podem ser muito úteis e têm sido muito utilizadas para monitorar a exposição ocupacional, mas é preciso utilizar medidas seriadas e correção pela creatinina. ${ }^{1}$ A excreção urinária de chumbo após administração de um quelante foi utilizada como índice de carga corpórea de chumbo. Entretanto, essa medida de chumbo na urina após a administração de quelante parece refletir apenas a mobilização de chumbo de tecidos moles e osso trabecular, não refletindo a carga corpórea total. ${ }^{2} \mathrm{O}$ plasma é o marcador que teoricamente reflitiria as concentrações de chumbo importantes do ponto de vista dos efeitos nos diferentes tecidos, entretanto, só muito recentemente começaram a surgir trabalhos com medidas confiáveis de chumbo no plasma feitas por Espectrometria de Massas com Plasma Indutivamente acoplado (ICP-MS). O plasma tem também um grande problema, que é a dificuldade de se coletar amostras de plasma sem hemólise. Como a concentração de chumbo no plasma é $1 \%$ daquela do sangue total,
1. Docente. Faculdade de Odontologia de Ribeirão Preto - USP.

2. Cirurgiã-Dentista, Especilista em Odontopediatria, Doutoranda pelo Programa de Pós-Graduação em Odontopediatria da FORP/USP.

3. Cirurgiã-Dentista, Especilista em Odontopediatria, Doutoranda pelo Programa de Pós-Graduação em Biologia Buco-Dental da FOP/UNICAMP.
Correspondência:

Profa. Dra. Raquel F. Gerlach Faculdade de Odontologia de Ribeirão Preto, FORP/USP Avenida do Café, S/N 14040-904 - Ribeirão Preto, SP Fone: 16-3602-4065 / Fax: 16-3602-4102 e-mail: rfgerlach@forp.usp.br

Artigo recebido em 26/08/2009 Aprovado em 08/12/2009 
qualquer contaminação com o chumbo das hemácias irá ter conseqüências dramáticas para os resultados.

Pode-se encontrar na literatura o uso de biomarcadores de efeito, sendo estes principalmente produtos da biossíntese do heme que se formam em maiores quantidades quando as enzimas que participam da via da síntese do heme estão inibidas pelo chumbo. Esses biomarcadores de efeitos foram vantajosos no passado para medir chumbo mais facilmente em indivíduos com alta exposição a chumbo, principalmente pelo menor custo das medidas e ausência de problemas com contaminação das amostras por chumbo exógeno. Estes biomarcadores de efeito ainda podem ser úteis para aferir rapidamente exposições a altas doses de chumbo ( $>25 \mu \mathrm{g} / \mathrm{dL}$ ). Entretanto, quando o chumbo está presente em baixas doses no sangue, esses marcadores deixam de ser úteis, e as medidas diretas de chumbo são mais adequadas. ${ }^{3}$

Nesta revisão iremos nos concentrar em sangue, osso e dentes como biomarcadores de exposição a chumbo. O sangue é sem dúvida o biomarcador mais utilizado e o único para o qual há conhecimento acumulado na literatura, formas de medidas fidedignas e também uma noção bem estabelecida de suas limitações. Entretanto, as medidas cumulativas mais aceitas hoje são as medidas de chumbo no osso in vivo para adultos, enquanto os dentes permitem a comparação de indivíduos ou grupos de indivíduos quanto à quantidade de chumbo, o que é particularmente interessante para detectar crianças expostas a quantidades excessivas de chumbo.

Como apontado por Bergdahl e Skerfving, ${ }^{2}$ entre os atributos que se precisa avaliar em um biomarcador de exposição a chumbo estão: 1) a acurácia e precisão analítica, 2) custo, 3) as questões práticas de uso (coleta por ex), 4) o que o biomarcador reflete, 5) a relação com exposição e 6) a relação com efeitos. Iremos discutir os diferentes biomarcadores à luz destes atributos a seguir.

\section{Sangue}

Em termos de acurácia e precisão analítica, o sangue é um dos biomarcadores mais vantajosos (leiase sempre sangue total para medidas de chumbo no sangue, pois $99 \%$ do chumbo no sangue está nas hemácias). O uso de medidas de chumbo no sangue é amplamente utilizado como teste laboratorial para aferir o grau de exposição, para estudos epidemiológicos e também para determinar o grau de exposição de cri- anças em vários estados dos EUA. ${ }^{4}$ Há experiência acumulada de cerca de 40 anos de uso de chumbo no sangue total e descrições amplamente aceitas dos protocolos de medidas analíticas. Nos EUA e em alguns outros países, há vários laboratórios governamentais e particulares que fornecem resultados fidedignos de medidas de chumbo no sangue. No Brasil há um número relativamente pequeno de laboratórios que faz medidas de chumbo no sangue. As determinações de chumbo no sangue normalmente são feitas por Espectrometria de Absorção Atômica com Forno de Grafite (GF-AAS), havendo métodos padronizados para sangue. ${ }^{5} \mathrm{O}$ limite de detecção descrito para esta técnica está torno de $1 \mu \mathrm{g} / \mathrm{dL}$ e são descritas variações entre medidas da mesma amostra de cerca de $5 \%$, o que é considerado uma variação pequena. ${ }^{2}$ É fundamental que os laboratórios que fazem medidas de chumbo de amostras clínicas de sangue participem de programas de calibração inter-laboratorial de chumbo no sangue (existem diversos em vários países, como por ex. o programa da Grã-Bretanha, o United Kingdom National External Quality Assessment Service, UKNEQAS, e o Programa de Ensaio de Proficiência para chumbo em sangue, PEP-Pb-s, coordenado pelo Instituto Adolfo Lutz, São Paulo, SP). Para determinar chumbo, os laboratórios precisam adotar medidas de rigoroso controle de qualidade e também usar amostras de referência contendo quantidades conhecidas de chumbo durante as análises. ${ }^{5}$

Há grande variação nos custos das medidas de chumbo no sangue. Bergdahl \& Skerfving ${ }^{2}$ avaliaram que o custo de uma medida de chumbo no sangue varia entre $\$ 4,00$ e $\$ 64,00$ dólares americanos. Claro que o uso de métodos bem estabelecidos, e rotina de grande número de medidas tende a diminuir os custos, mas o custo alto e a falta de rotina para medidas de chumbo no sangue ainda são problemas que dificultam o uso amplo deste teste importante para avaliar exposição a chumbo no Brasil.

Do ponto de vista da coleta, é relativamente simples a coleta de sangue, que normalmente é feita por punção venosa no braço utilizando-se um tubo BD trace metal free contendo Na2EDTA ou heparina ou, no caso de crianças pequenas, o ideal é utilizar o tubo BD plástico para coleta de $3 \mathrm{~mL}$ (Vacutainer Plus Low Lead, Becton Dickinson Vacutainer Tube Systems) contendo K2EDTA e produzido especificamente para testar chumbo em crianças. ${ }^{6}$ Estes tubos são certificados e apresentam menos do que $0,25 \mu \mathrm{g} / \mathrm{dL}$ de chumbo. Após a coleta, o tubo pode ser guardado na 
geladeira por alguns meses até o momento da determinação de chumbo. Para medidas de chumbo em crianças nos EUA, coleta de sangue capilar feita num dedo da mão foi utilizada por certo tempo. Este método é muito sujeito a contaminação, ${ }^{7}$ e não traz muitas vantagens do ponto de vista do desconforto da criança, e por isso não é mais usado.

O chumbo no sangue pode refletir a exposição recente a chumbo do meio externo assim como o chumbo mobilizado do compartimento ósseo. ${ }^{4}$ Geralmente a maior parte do chumbo no sangue reflete a exposição recente (semanas a meses), uma vez que o chumbo tem uma meia vida relativamente curta no sangue: cerca de um mês. ${ }^{8,9} \mathrm{O}$ termo meia vida é muitas vezes substituído por "tempo de residência" em determinado compartimento do organismo para descrições sobre chumbo. ${ }^{1}$ Entretanto, há situações em que a maior parte do chumbo no sangue pode não refletir a exposição a chumbo do meio externo, mas sim o chumbo do osso, como é o caso em situações caracterizadas por altas taxas de remodelamento ósseo, como o crescimento, a gravidez e o período pós-menopausa. ${ }^{4}$

Existe uma forma de utilizar medidas repetidas de sangue para ter uma medida cumulativa, mesmo que as medidas de sangue não tenham sido obtidas de forma sistemática a intervalos regulares. Esse índice é chamado de Índice cumulativo de chumbo no sangue (Cumulative Blood Lead Index, CBLI) e se baseia no uso da área sob a curva de chumbo no sangue traçada para um determinado indivíduo para calcular a exposição, assim se tem como parâmetro as concentrações e o tempo. ${ }^{4} \mathrm{O}$ cálculo da área sob a curva é feito simplesmente pela plotagem dos valores no eixo y e do tempo no eixo x em papel milimetrado e o cálculo da área sob a curva, que resulta em $\mu$ g-anos/dL. Esse índice é muito importante, principalmente para medir exposições ocupacionais quando não há possibilidade de fazer medidas de osso, pois foi demonstrado haver forte correlação entre o CBLI e as medidas de chumbo no osso cortical. ${ }^{10}$ No caso do Brasil, onde não há ainda um equipamento para medidas diretas in vivo de chumbo no osso, o uso de CBLI pode ser uma alternativa para estimar melhor os riscos da exposição crônica em trabalhadores.

O uso do CBLI e das medidas de chumbo no osso cortical foram fundamentais para as associações demonstradas em anos recentes entre chumbo e algumas doenças crônicas como hipertensão ${ }^{11}$ e diminuição da função cognitiva em adultos. ${ }^{12}$ Resultado do grande aumento nas evidências de doenças de adul- tos associadas a níveis moderados de chumbo acumulado é a sugestão de mudança nos níveis aceitos nos EUA para exposição ocupacional a chumbo. ${ }^{13}$

Do ponto de vista da relação entre medidas de chumbo no sangue com efeitos, já é bem aceito na literatura que há bastante variabilidade na suscetibilidade individual a efeitos para uma mesma concentração de chumbo no sangue. ${ }^{2}$ Isso poderia decorrer de variações individuais em vários polimorfismos genéticos, que parecem caracterizar subgrupos populacionais particularmente suscetíveis aos efeitos do chumbo. Além disso, algumas doenças crônicas como a diabete tipo II tem sido associadas a piores efeitos em concentrações mais baixas de chumbo. ${ }^{13}$

\section{Osso}

Conforme discutido em outros artigos deste simpósio, o chumbo que é absorvido ao longo da vida de um indivíduo se acumula no osso (cerca de $95 \%$ do chumbo em adultos). ${ }^{4}$ Normalmente a quantidade de chumbo só aumenta no osso ao longo da vida.

Em decorrência do acúmulo no osso, as medidas de chumbo no osso há algum tempo passaram a ser vistas como as melhores medidas de exposição cumulativa a chumbo. ${ }^{4} \mathrm{Em}$ trabalhos em animais, podese fazer a determinação direta em ossos após a morte do animal, e esta técnica é também utilizada para verificar a quantidade de chumbo em pessoas após a morte e em ossos de centenas de anos. Nestes casos o chumbo é medido por técnicas quantitativas bem estabelecidas, como GF-AAS, voltametria anódica ou ICP-MS. Um dos dois elementos mais abundantes do osso (fósforo e cálcio) é também determinado, o que permite a expressão dos resultados em micrograma de chumbo por grama de osso $(\mu \mathrm{g} / \mathrm{g}$, normalmente de osso seco). As medidas em osso seco apresentam variação de $20 \%$ a mais na concentração de chumbo em comparação com medidas obtidas in vivo.

Indivíduos não expostos a chumbo no ambiente de trabalho apresentam cerca de $20 \mu \mathrm{g} / \mathrm{g}$ de chumbo no osso, enquanto trabalhadores expostos podem apresentar $100 \mu \mathrm{g} / \mathrm{g} .{ }^{2}$ Trabalhadores com esta concentração no osso podem apresentar $16 \mu \mathrm{g} / \mathrm{dL}$ de chumbo no sangue pela mobilização contínua de chumbo do osso. ${ }^{1}$ No caso de mulheres com cerca de $50 \mu \mathrm{g} / \mathrm{g}$ de chumbo no osso, estas poderão apresentar medidas no sangue de $8 \mu \mathrm{g} / \mathrm{dL}$, sem considerar o aumento ainda maior nos períodos de intenso remodelamento ósseo, quando mais chumbo é mobilizado do osso. ${ }^{1}$ 
A determinação de chumbo em ossos em seres humanos vivos se tornou possível com o desenvolvimento de uma técnica chamada fluorescência de raio $\mathrm{X}$ para uso in vivo. A fluorescência de raios X (X-ray fluorescence, XRF) já era amplamente utilizada para análises químicas de materiais, com a grande vantagem de permitir a determinação de vários elementos ao mesmo tempo. A adaptação do uso de XRF para uso in vivo começou em meados de $1980,{ }^{14} \mathrm{e}$ os primeiros resultados de estudos populacionais utilizando XRF aparecem em meados da década de $1990 .{ }^{4}$

A fluorescência é um fenômeno óptico que ocorre pela emissão de energia decorrente do deslocamento de elétrons de orbitais mais externos para os mais internos. ${ }^{15}$ Por exemplo: do orbital L para o orbital $\mathrm{K}$. Esse deslocamento acontece quando uma energia superior àquela do potencial de ionização de um átomo é aplicada, do que resulta que um elétron de uma camada mais interna (K) seja deslocado para um orbital mais externo, resultando numa estrutura atômica instável, e no deslocamento de um elétron de orbitais mais externos para o mais interno que se tornou "incompleto", com liberação de energia igual à diferença de energia entre os 2 orbitais. Como essas energias são muito características de orbitais de diferentes elementos, a análise muitas vezes pode ser multielementar, dependendo da capacidade do detector. Chumbo, cálcio e muitos outros elementos têm essa característica de emitir fluorescência quando excitados com energia adequada. A fonte de energia usada para produzir fótons fluorescentes do orbital $\mathrm{K}$ (por isso KXRF) característicos do chumbo para medidas em osso tem sido a radiação gama de baixa intensidade do cádmio109. ${ }^{4}$ Há também medidas in vivo feitas com LXRF (que usa medidas baseadas no deslocamento de elétrons da camada L), mas há menos trabalhos utilizando esta técnica.

O tempo de medida requerido para obter sinal adequado é no mínimo 30 minutos, e é fundamental que a área irradiada esteja totalmente imóvel. A quantidade de radiação emitida tem sido comparada àquela de uma tomada de radiografia odontológica pequena, o que é bastante aceitável do ponto de vista de riscos à saúde. ${ }^{4,15}$ Entretanto, autores que pesquisam melhorias nos instrumentos atuais de XRF apontam para o cuidado que se deve ter ao expor crianças, uma vez que a dose recebida por uma criança será muito maior do que a de um adulto em função da presença de medula óssea vermelha na tíbia da criança. ${ }^{16}$
Nesta técnica, a calibração entre medidas e calibração entre instrumentos é feita com modelos de gesso contendo quantidades conhecidas de chumbo. Entretanto padrões internacionais para calibração interlaboratorial (a exemplo dos de sangue) ainda não existem.

As medidas de chumbo feitas em ossos como a tíbia de cadáveres com concentrações de chumbo no osso entre 20 e $30 \mu \mathrm{g} / \mathrm{g}$ mostram desvios padrões de 3-4 $\mu \mathrm{g} / \mathrm{g},{ }^{17}$ o que é uma variação relativamente grande em comparação com técnicas para sangue. Outro problema sério é que comumente pessoas não expostas ocupacionalmente apresentam medidas negativas de chumbo no osso por XRF (o que naturalmente não é real, mas decorre do cálculo da medida baseado nos padrões de gesso), e assim um desafio atual é diminuir o limite de detecção mínimo por esta técnica. ${ }^{18}$

Além destes problemas, as medidas de chumbo no osso apresentam maior grau de incerteza se for maior a quantidade de tecido mole sobre o osso. ${ }^{18}$ Além disso, como as medidas dependem da quantidade de massa óssea, o índice de massa corporal pode ter efeito nas medidas, e o grau de incerteza aumenta muito em mulheres e crianças. ${ }^{4}$

Não há uma variação importante quando quantidades de chumbo de posições simétricas foram medidas. ${ }^{17}$ Há trabalhos que fizeram medidas em falange, patela, tíbia e calcâneo, sendo que as medidas de falange praticamente só são confiáveis em indivíduos expostos ocupacionalmente. ${ }^{2}$

As descrições mais frequientes são de chumbo na patela e na tíbia. A patela é um osso predominantemente trabecular, onde o tempo de residência do chumbo é de poucos anos. Já a tíbia é um osso predominantemente cortical, sendo as medidas feitas na região da diáfise, em que o tempo de residência do chumbo é de cerca de 30 anos. $^{1}$

Portanto, em termos de comparação com o sangue, as medidas de chumbo no osso não têm a mesma acurácia e precisão analíticas, e não podem ainda ser feitas em laboratórios comerciais ou governamentais. Há menos de uma dezena de equipamentos de XRF para medidas in vivo de chumbo no osso no mundo, e estes são utilizados para estudos epidemiológicos até o momento. ${ }^{4}$

Do ponto de vista da medida, o tempo de meia hora e a imobilidade dificultam a medida, que tem uma precisão razoável em homens adultos. Em mulheres e crianças a variabilidade das medidas é muito grande $\mathrm{e}$ uma boa porcentagem das medidas de pessoas não 
expostas ocupacionalmente costuma ser negativa. Apesar destes problemas, o uso de medidas de chumbo acumulado no osso, particularmente no osso cortical, e os dados de CBLI foram fundamentais para a demonstração de que maior exposição cumulativa a chumbo está associada a risco aumentado de doenças crônicas em populações adultas. ${ }^{13}$

\section{Dentes}

O chumbo se acumula nos dentes e nos ossos, mas as medidas de chumbo em dentes e ossos trazem informações distintas quanto à exposição a chumbo.

Os dentes podem representar uma alternativa potencialmente interessante do ponto de vista da coleta de amostras e da facilidade de medidas de amostras de dentes. Isso se torna mais importante quando lembramos que as medidas de chumbo no osso in vivo podem ser feitas em poucos laboratórios do mundo e que esse método ainda não está adequadamente desenvolvido para medidas em crianças. Além disso, enquanto os ossos são inacessíveis para medidas diretas por técnicas estabelecidas, os dentes de leite são perdidos e podem ser usados para medidas diretas. Mesmo os dentes presentes na boca permitem que sejam obtidas amostras superficiais para medir chumbo, sem haver dano para o sujeito da pesquisa. Finalmente, os dentes trazem informações sobre a exposição em crianças que só são conseguidas por várias medidas seriadas de sangue da mãe e da criança (o que tem um custo alto e dificuldades de coleta). Finalmente, as medidas de chumbo em amostras de dentes de leite muitas vezes representam a exposição a chumbo de um período precoce do desenvolvimento, quando os efeitos do chumbo são particularmente nocivos.

Para compreender a utilidade dos dentes como biomarcadores é preciso levar em conta a "cronologia" de formação e mineralização de cada grupo de dentes, além das características de cada tecido que compõe os dentes. ${ }^{19}$ Levando isso em conta, é possível identificar as partes dos dentes que registram a exposição em um período específico do passado e outras partes que trazem informação sobre a exposição acumulada de certo momento no passado até o momento em que o dente foi perdido.

Os trabalhos utilizando a dentina de dentes de leite como marcador da exposição passada e cumulativa a chumbo foram fundamentais para estabelecer que a exposição precoce a baixas doses de chumbo estava associada a perdas cognitivas e diminui- ção do desempenho escolar. ${ }^{20,21}$ Infelizmente em anos recentes houve um grande desinteresse pelo uso de dentes para medidas de chumbo, o que pode decorrer do uso de medidas seriadas de chumbo no sangue em estudos recentes ${ }^{22}$ e do grande número de informações inconsistentes sobre chumbo em dentes publicadas nos últimos anos em periódicos de diferentes áreas.

Aqui iremos ressaltar alguns aspectos biológicos e metodológicos a considerar ao analisar trabalhos de chumbo em dentes, mostrando a seguir as informações que já tivemos no Brasil sobre exposição de crianças a chumbo aferida a partir de amostras de dentes.

Os dentes têm uma coroa (que é visível na boca) e uma raiz (Figura 1). A coroa é revestida externamente pelo esmalte, enquanto a raiz é revestida pelo cemento. A dentina é o tecido mineralizado subjacente ao esmalte e ao cemento. Internamente está localizada a polpa, tecido conjuntivo não mineralizado, que nutre a dentina. ${ }^{19,23}$ Os dentes de leite são perdidos sem a maior parte da raiz (Figura $1 \mathrm{~A}$ e B).

Dentina, cemento e osso são tecidos mineralizados bastante semelhantes do ponto de vista bioquímico: os três têm uma matriz orgânica majoritariamente constituída de colágeno tipo I, o mineral (hidroxiapatita) se precipita sobre esse arcabouço orgânico, e a quantidade de mineral fica em torno de $70 \%$ de peso. Estes três tecidos tem respostas semelhantes a estímulos hormonais e normalmente uma camada contínua de células separa o compartimento mineral (a matriz) do líquido tecidual do restante do organismo. Há também muitas semelhanças entre estes três tecidos quanto ao acúmulo de chumbo, sendo a mais óbvia a quantidade relativa de chumbo encontrada, que é da mesma ordem de magnitude. Há também uma diferença fundamental entre estes tecidos a considerar para comparar medidas de chumbo: enquanto o osso é um tecido que se remodela constantemente (a sua estrutura é continuamente modificada), a dentina praticamente não se remodela. Portanto, na dentina o chumbo será incorporado enquanto esta estiver sendo depositada, enquanto no osso o chumbo será continuamente depositado, dependendo da taxa de remodelamento ósseo.

Uma camada espessa de dentina é produzida enquanto o dente se forma e até ele surgir na boca (erupcionar) e tomar o seu lugar. Essa dentina é produzida com maior velocidade e se chama dentina primária. Depois que o dente já erupcionou toda a sua 


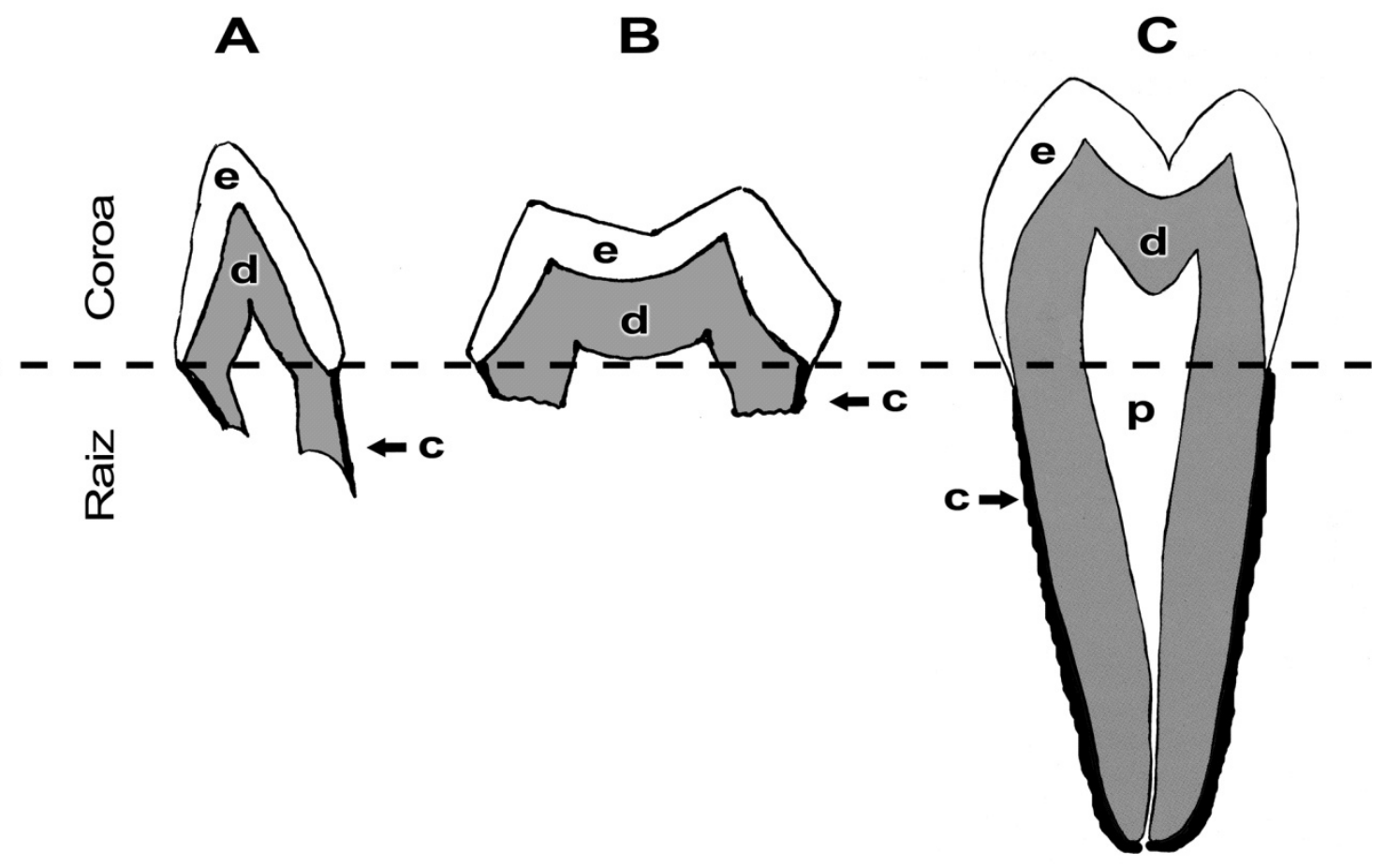

Figura 1. Desenho esquemático de dentes de leite $(A$ e $B)$ e de um dente permanente $(C)$. A linha tracejada indica onde acaba a coroa $e$ inicia a raiz. e: esmalte, d: dentina, p: polpa, c: cemento. Notar que os dentes de leite estão sem a maior parte da raiz.

raiz está formada as células da polpa junto à dentina continuam a produzir dentina durante toda a vida do dente, mas numa velocidade muito mais baixa. Essa camada é a "dentina secundária", que tem grande importância aqui porque muitos trabalhos descrevem a quantidade de chumbo acumulada nesta camada (em muitos trabalhos no exterior ela foi chamada de dentina circumpulpar, mas é um termo que não iremos usar aqui). A dentina secundária e o cemento são tecidos que apresentam as maiores quantidades de chumbo em comparação com a dentina primária e osso. ${ }^{24}$

As crianças tem 20 dentes de leite, que são perdidos entre os 6-12 anos, nascendo a seguir os dentes permanentes (que são 32). O período de formação deste grande número de dentes começa na vida intra-uterina e vai até os 15-18 anos. Por isso, dentes têm sido usados como registro de eventos que ocorrem ao longo da vida de um indivíduo e que não ficam registrados definitivamente em outros tecidos, pois estes normalmente se modificam ao longo da vida. Esse registro é muito usado por antropólogos..$^{25}$

Entre os eventos registrados nos dentes está o momento do nascimento, que aparece como uma linha no esmalte e na dentina, a linha neonatal. ${ }^{23}$ Essa linha pode ser usada para se conhecer o que acontecia antes e depois do nascimento. A referência a esta linha neonatal é utilizada em trabalhos de chumbo em dentes, mas o seu significado é pouco compreendido: enquanto na dentina ela realmente separa partes da dentina que mineralizaram antes e depois do nascimento, no esmalte isso não ocorre. Essa diferença decorre da diferença no processo de mineralização de dentina e esmalte. Como dentina é depositada em incrementos microscópicos e não se remodela, o que foi depositado antes do nascimento é distinto do que foi depositado após o nascimento.

Com base nessas informações e na época em que a dentina primária é depositada nos diferentes dentes, é possível usar diferentes partes da dentina primária para aferir a exposição a chumbo num certo momento do passado. Além disso, a dentina secundária de dentes de leite trará a informação sobre o chumbo acumulado entre a época que um determinado dente entrou em função (por ex. 12 meses de vida no caso dos incisivos centrais decíduos) e a época em que estes foram perdidos (por ex. 6 ou 7 anos). Essas informações foram cuidadosamente obtidas no passado. ${ }^{20,21,24,26}$ 
Além dos trabalhos com escolares para aferir a exposição passada cumulativa a chumbo, a dentina também é muito útil para estimar a exposição de populações antigas, pois ela está relativamente protegida do meio externo. ${ }^{24}$ A comparação de medidas de chumbo na dentina secundária de dentes permanentes de mais de 5000 anos com dentes de pessoas que viveram na Dinamarca na Idade Média e atualmente revelaram que a exposição a chumbo era muito baixa há 5000 anos (mediana: $2 \mu \mathrm{g} / \mathrm{g}$ de chumbo na dentina), chegando a $100 \mu \mathrm{g} / \mathrm{g}$ na Idade Média, enquanto atualmente dinamarqueses adultos não expostos apresentavam cerca de $23 \mu \mathrm{g} / \mathrm{g}$ de chumbo na dentina. $\mathrm{O}$ mesmo padrão de mudanças foi visto quando se usou a razão $\mathrm{Pb} / \mathrm{Ca}$ para esta comparação, e estes dados concordam o aumento na concentração de chumbo verificado como consequiência das atividades humanas. As menores concentrações de chumbo recentes na Dinamarca estão de acordo com a relativamente baixa exposição a chumbo vista naquele país. ${ }^{24}$

Apesar de sua importância e algumas vantagens, a dentina só pode ser coletada em dentes que já foram perdidos. Para conseguir um fragmento de dentina, é preciso fraturar, moer ou tirar uma fatia de um dente, processo laborioso que facilmente leva à contaminação da amostra. Conseguido o fragmento, este é dissolvido em ácido para as medidas de chumbo e cálcio ou fósforo, o que permite expressar o resultado em $\mu \mathrm{g}$ de chumbo/g de dentina $(\mu \mathrm{g} / \mathrm{g})$.

Acreditamos que entre as principais razões para poucos trabalhos recentes usarem dentes como biomarcadores estão a dificuldade técnica para se obter fragmentos de dentina e a falta de trabalhos sobre a correlação entre as concentrações de chumbo na dentina e as quantidades de chumbo no sangue ao longo do tempo.

O esmalte, por outro lado, não tem nenhuma semelhança com a dentina e o osso do ponto de vista da mineralização. ${ }^{23} \mathrm{~A}$ mineralização do esmalte não é aposicional, como a da dentina. $\mathrm{O}$ esmalte maduro que encontramos nos dentes na boca é o tecido mais mineralizado do nosso corpo ( $95 \%$ de mineral em peso e menos de $1 \%$ de proteínas) e se mineraliza por meses a anos depois da matriz ter sido depositada a partir do contínuo influxo de íons através da superfície. ${ }^{27} \mathrm{~A}$ compreensão de como o esmalte se mineraliza é bastante recente. ${ }^{28}$ É essa forma distinta de mineralização que resulta no acúmulo de certos íons na superfície do esmalte, e sua distribuição muito distinta no esmalte e na dentina. Entre os íons encontrados em maiores concentrações na superfície estão flúor, chumbo, cádmio, zinco e estrôncio. ${ }^{29,30,31}$

Estudos com número razoável de crianças mostram que o esmalte dos dentes pode ser muito útil para a obtenção de uma amostra superficial, na qual o chumbo pode ser medido fidedignamente, permitindo encontrar crianças excessivamente expostas ou regiões de maior ou menor contaminação por chumbo. ${ }^{30,32-36}$ A grande vantagem do uso de esmalte é que a amostra pode ser obtida in vivo em menos de 1 minuto, por um teste indolor e que não causa comprometimento ao dente. ${ }^{37}$

Nós realizamos alguns trabalhos utilizando coletas in vivo de esmalte superficial de dentes de leite para verificar a quantidade de chumbo em escolares. 35,36,37 Esta técnica surgiu em 1956, para conseguir amostras superficiais de dentes extraídos. ${ }^{29}$ Como dito na Introdução deste Simpósio, nesta época ainda não se usavam técnicas limpas para determinação da maioria dos elementos, e assim as concentrações de milhares de $\mu \mathrm{g} / \mathrm{g}$ de chumbo no esmalte superficial encontradas em meados da década de $1970^{38}$ eram vistas com desconfiança, uma vez que na dentina a quantidade máxima na maioria dos trabalhos fica em torno de $40 \mu \mathrm{g} / \mathrm{g}$ e pouco ainda se sabia sobre as diferenças na forma distinta de mineralização do esmalte e da dentina.

A partir da análise dos dados obtidos, é possível perceber crianças e populações de crianças com concentrações bem mais altas de chumbo, o que é muito característico quando se observam amostras coletadas de crianças que sempre residiram nas proximidades de uma fábrica de baterias, como é o caso da AJAX, em Bauru, SP. Isso ficou bem claro em um trabalho nosso em que comparamos as quantidades de chumbo em dente de leite coletados em 7 Escolas Municipais de Educação Infantil (4-6 anos) e nas crianças que moravam perto da Fábrica da AJAX. ${ }^{39}$ Uma análise preliminar sugere que concentrações em torno de $600 \mu \mathrm{g} / \mathrm{g}$ de chumbo no esmalte indiquem exposição excessiva quando esta quantidade estiver na entre 3 e 6 um da superfície externa do esmalte (Figura 2).

Esse trabalho, assim como os anteriores ${ }^{30,32-36}$ e o estudo recentemente realizado em Ribeirão Preto em 2008, com coletas de sangue, saliva e amostras de esmalte de 444 escolares de 6-8 anos, ${ }^{40,41}$ mostram que algumas crianças apresentam exposição excessivamente alta, mesmo em regiões sem contaminação notória por chumbo descrita. Esses achados não cau- 


\section{\% de crianças}

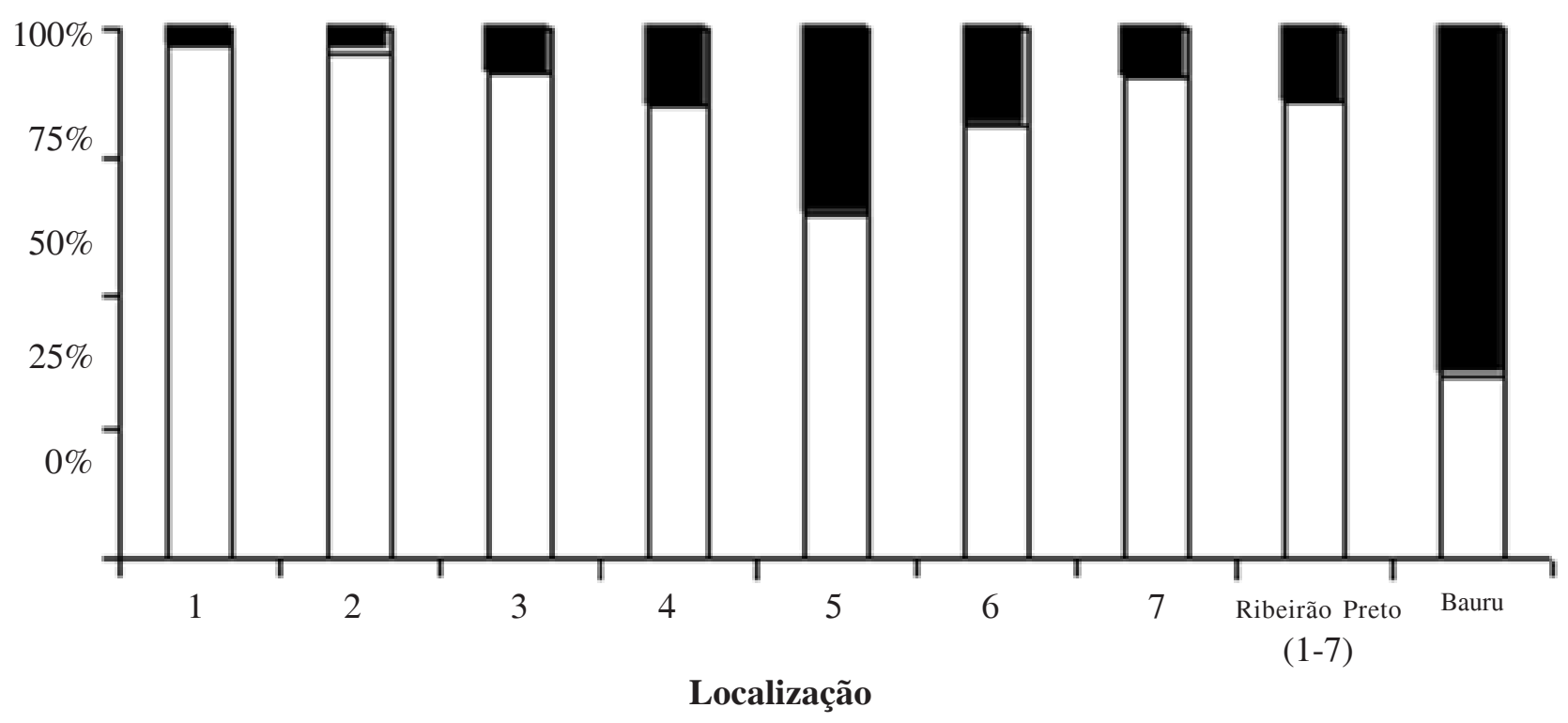

Figura 2. Porcentagens de crianças distribuídas de acordo com valores de chumbo 600 ou $<600 \mu \mathrm{g} / \mathrm{g}$ em amostras de esmalte superficial obtidas in vivo em 7 Escolas Municipais de Ribeirão Preto, SP $(n=186)$ e nas cercanias da Fábrica de Baterias AJAX, em Bauru, SP $(n=20)(2004)$. Notar a maior porcentagem de crianças com chumbo de chumbo $600 \mu \mathrm{g} / \mathrm{g}$ na Escola 5 de Ribeirão Preto em comparação com as demais. Figura reproduzida de Environmental Research 107: 264-70, 2008, com permissão da Editora Elsevier.

sam espanto, uma vez que o chumbo é amplamente utilizado para vários fins e seu controle em vários países não é muito rígido (e as formas de monitoramento na população em geral muitas vezes inexistentes). Os dados indicam que é preciso estudar cuidadosamente a prevalência da exposição indevida, as fontes de chumbo e estimar as perdas para a população, para que se possa prevenir completamente a exposição excessiva.

\section{Conclusões}

As medidas de chumbo no sangue total são as mais adequadas do ponto de vista da acurácia e precisão analítica e das informações acumuladas ao longo das últimas décadas sobre a relação de medidas de chumbo no sangue com efeitos. Recentemente medidas no osso in vivo por XRF se tornaram possíveis e permitiram trabalhos epidemiológicos com centenas de indivíduos, os quais mostram que índices cumulativos (como medidas repetidas de sangue ou medidas no osso) são essenciais para verificar os efeitos do chumbo em adultos. Os dentes deixaram de ser usados nas últimas décadas, mas podem ser uma alternativa importante para verificar exposição excessiva em crianças, especialmente em países em que medidas repetidas no sangue não são feitas. Para utilizar dentes é fundamental prestar atenção aos diferentes tecidos e aos cuidados com contaminação exógena. As informações de diferentes biomarcadores de exposição a chumbo são distintas e muitas vezes complementares.

\section{ABSTRACT:}

This article aims at describing some of the most important biomarkers of exposure to lead, showing its advantages and limitations, as well as how they relate to present, past or cumulative exposure to lead. This basic knowledge is essential for the comprehension of the effects of lead, the difficulties of the diagnosis of excessive exposure, and the problem of exposure to low levels of lead. The biomarkers of exposure discussed in depth in this article are blood, bone, and teeth.

keywords: Lead. Biomarkers, Pharmacological. Whole Blood. Bone. Tooth. 


\section{Referências Bibliográficas}

1. Barbosa F Jr, Tanus-Santos JE, Gerlach RF, Parsons JP. A critical review of biomarkers used for monitoring human exposure to lead: advantages, limitations, and future needs. Environ Health Perspect. 2005;113:1669-74.

2. Bergdahl IA, Skerfving S. Biomonitoring of lead exposure alternatives to blood. J Toxicol Environ Health. 2008;71:123543.

3. Parsons PJ, Reilly AA, Hussain A. Observational study of erythrocyte protoporphyrin screening test for detecting low lead exposure in children: impact of lowering the blood lead action threshold. Clin Chem. 1991; 37:216-25.

4. Hu H, Shih R, Rothemberg S, Schwartz BS. The epidemiology of lead toxicity in Adults: measuring dose and consideration of other methodologic issues. Environ Health Perspect. 2007; 115:455-62.

5. Parsons PJ, Slavin W. A rapid Zeeman graphite furnace atomic absorption spectrometric method for the determination of lead in blood. Spectrochim Acta, B 1993; 48:925-39.

6. Esernio-Jenssen D, Busch V, Parsons JP. Evaluation of VACUTAINER PLUS Low Lead Tubes for blood lead and erythrocyte protophorphirin testing. Clin Chem. 1999; 45:148-50.

7. Parsons PJ, Reilly AA, Esernio-Jenssen D. Screening children exposed to lead: an assesment of the capilarry blood lead fingerstick test. Clin Chem. 1997;43: 302-11.

8. O’Flaherty EJ. Physiologically based models for bone-seeking elements. V: Lead absorption and disposition in children. Toxicol Appl Pharmacol. 1995;131: 297-308.

9. Rabinowitz MB, Wetherill GW, Koopple JD. Kinetic analysis of lead metabolism in healthy humans. J Clin Invest.1976;58: 260-70.

10. Roels H, Konings J, Green S, Bradley D, Chettle D, Lauwerys $R$. Time-integrated blood lead concentration is a valid surrogate for estimating the cumulative lead dose assessed by tibial lead measurement. Environ Res. 1995; 69:75-82.

11. Shih RA, Hu H, Weisskopf M, Schwartz BS. Cumulative lead dose and cognitive function in adults : a review of studies that measured both blood and bone lead. Environ Health Perspect. 2007;115:483-92.

12. Navas-Acien A, Gualler E, Silbergeld EK, Rothemberg SJ. Lead exposure and cardiovascular disease - a systematic review. Environ Health Perspect. 2007;115:472-82.

13. Schwartz BS, Hu H. Adult lead exposure: time for change. Environ Health Perspect. 2007;115:451-4.

14. Hu H, Rabinowitz M, Smith D. Bone lead as a biological marker in epidemiologic studies of chronic toxicity: Conceptual paradigms. Environ Health Perspect. 1998;106:1-8.

15. Todd AC, Chettle DR. In vivo X-ray fluorescence of lead in bone: review and current issues. Environ Health Perspect. 1994;102:172-7.

16. Nie H, Chettle D, Luo L, O’Meara J. Dosimetry study for a new in vivo X-ray fluorescence (XRF) bone lead measurement system. Nucl Instr and Meth in Phys Res. B 2007; 263: 22530

17. Aro A, Amarasiriwardena C, Lee ML, Kim R, Hu H. Validation of $\mathrm{K}$ x-ray fluorescence bone lead measurements by inductively coupled plasma mass spectrometry in cadaver legs. Med Phys. 2000; 27:119-23.
18. Ahmed N, Fleming DEB, Wilkie D, O'Meara JM. Effects of overlying soft tissue on X-ray fluorescence bone lead measurement uncertainty. Radiation Physics and Chemistry. 2006; 75:1-6.

19. Nanci A. Histologia oral de Ten Cate: desenvolvimento, estrutura e função. 7ª Ed. Rio de Janeiro (RJ): Elsevier; 2008.

20. Needleman HL, Gunnoe C, Leviton A, Reed R, Peresie H, Maher C, Barret P. Deficits in psychologic and classroom performance of children with elevated dentine lead levels. $\mathrm{N}$ Engl J Med. 1979; 300: 689-95.

21. Hansen ON, Trillingsgaard A, Beese I, Lyngbye T, Grandjean P. A neuropsychological study of children with elevated dentine lead level: assessment of the effect of lead in different eosio-economic groups. Neurotoxicol Teratol. 1989;11:20513.

22. Canfield RL, Henderson CRJ, Cory-Slechta DA, Cox C, Jusko TA, Lanphear BP. Intellectual impairment in children with blood lead concentrations below $10 \mu \mathrm{g}$ per deciliter. $\mathrm{N}$ Engl J Med. 2003; 348: 1517-26.

23. Avery JK (ed), Steele PF (assoc. ed): Oral development and histology. Third Edition, Thieme, New York: 2002.

24. Grandjean P, Jorgensen PJ. Retention of lead and cadmium in prehistoric and modern human teeth. Environ Res.1990;53:615.

25. Dean MC. Tooth microsctructure tracks the pace of human life-history evolution. Proc R Microsc Soc. B. 2006; 273 2799-808.

26. Needleman HL, Tuncay OC, Shapiro IM. Lead levels in deciduous teeth of urban and suburban American children. Nature (Lond.) 1972; 235: 111-2.

27. Gerlach RF, Line SRP. Ameloblastos. In: Carvalho HF, CollaresBuzato CB (editores). Células: uma abordagem multidisciplinar. $1^{\underline{a}}$ ed. Barueri (SP): Manole; 2005. p. 95-106.

28. Smith CE. Cellular and chemical events during enamel maturation. Crit Rev Oral Biol Med. 1998; 9:128-36.

29. Brudevold F, Steadman LT. The distribution of lead in human enamel. J Dent Res. 1956; 35:43.

30. Cleymaet R, Retief DH, Quartier E, Slop D, Coomans D, Michotte Y. A comparative study of the lead and cadmium content of surface enamel of Belgian and Kenyan children. Sci Total Environ. A 1991;104:175-89.

31. Robinson C, Kirkham J, Brookes SJ, Roger CS. Chemistry of mature enamel (Chapter 8). In: Robinson C, Kirkham J, Shore $R$ (eds). Dental enamel: formation to destruction. 1st ed. London, CRC Press, 1995.

32. Cleymaet R, Quartier E, Slop D, Retief DH, Smeyers-Verbeke $\mathrm{J}$, Coomans D. Model for assessment of lead content in human surface enamel. J Toxicol Environ Health. B 1991; 32:11127

33. Cleymaet R, Bottenberg P, Retief DH, Slop D, Michotte $\mathrm{Y}$, Coomans D. In vivo use of a dual acid etch biopsy for the evaluation of lead profiles in human surface enamel. Caries Res. 1991c; 25:256-63.

34. Cleymaet R, Collys K, Retief DH, Michotte Y, Slop D, Taghon $\mathrm{E}$, Maex W, Coomans. Relation between lead in surface tooth enamel, blood, and saliva from children residing in the vicinity of a non-ferrous metal plant in Belgium. $\mathrm{Br} \mathrm{J}$ Ind Med. 1991d; 48:702-9.

35. Gomes VE, Sousa MLR, Barbosa F, Krug FJ, Saraiva MCP, Cury JA, Gerlach RF. In vivo studies on lead content of de- 
ciduous teeth superficial enamel of pre-school children. Sci Total Environ. 2004; 320: 25-35.

36. Costa de Almeida GR, Pereira Saraiva Mda C, Barbosa F Jr, Krug FJ, Cury JA, Rosário de Sousa M da L, Rabelo Buzalaf MA, Gerlach RF. Lead contents in the surface enamel of deciduous teeth sampled in vivo from children in uncontaminated and in lead-contaminated areas. Environ Res. 2007; 104: $337-45$.

37. Costa de Almeida GR, Molina GF, Meschiari CA, Barbosa de Sousa F, Gerlach RF. Analysis of enamel microbiopsies in shed primary teeth by Scanning Electron Microscopy (SEM) and Polarizing Microscopy (PM). Sci Total Environ. 2009; 407: 5169-75.

38. Brudevold F, Redá A, Aasenden R, Bakhos Y. Determination of trace elements in surface enamel of human teeth by a new biopsy procedure. Arch Oral Biol. 1975; 20: 667-73.
39. De Almeida GR, de Souza Guerra C, Tanus-Santos JE, Barbosa FJr, Gerlach RF. A plateau detected in lead accumulation in subsurface deciduous enamel from individuals exposed to lead may be useful to identify children and regions exposed to higher levels of lead. Environ Res. 2008; 107: 264-70.

40. Costa de Almeida, GR. Utilização de biomarcadores de dose interna para avaliação da exposição ao chumbo e suas correlações com anemia e polimorfismos genéticos em crianças residentes em uma região supostamente não contaminada. [Tese de Doutorado]. Campinas: Universidade Estadual de Campinas, 2009.

41. Costa de Almeida GR, de Freitas Tavares CF, de Souza AM, Sampaio de Sousa T, Rodrigues Funayama CA, Barbosa F Jr, Tanus-Santos JE, Gerlach RF. Whole blood, serum, and saliva lead concentrations in 6- to 8-year-old children. Sci Total Environ. 2010; 408:1551-56. 